High field and Coulomb interaction effects on spin injection in degenerate semiconductors

This article has been downloaded from IOPscience. Please scroll down to see the full text article.

2004 Semicond. Sci. Technol. 19 S383

(http://iopscience.iop.org/0268-1242/19/4/126)

View the table of contents for this issue, or go to the journal homepage for more

Download details:

IP Address: 128.206.162.204

The article was downloaded on 02/07/2010 at 15:26

Please note that terms and conditions apply. 


\title{
High field and Coulomb interaction effects on spin injection in degenerate semiconductors
}

\author{
Irene D'Amico $^{1}$ and Giovanni Vignale ${ }^{2}$ \\ ${ }^{1}$ Institute for Scientific Interchange, via Settimio Severo 65, I-10133 Torino, \\ Italy \\ ${ }^{2}$ Department of Physics, University of Missouri, Columbia, MO 65211, USA \\ E-mail: damico@isiosf.isi.it
}

Received 28 July 2003

Published 12 March 2004

Online at stacks.iop.org/SST/19/S383 (DOI: 10.1088/0268-1242/19/4/126)

\begin{abstract}
We analyse the combined effect of high electric field and Coulomb interaction on spin transport in semiconductors. We concentrate on the experimentally relevant regime characterized by $T \lesssim T_{F}$ (intermediate to degenerate), where $T_{F}=\varepsilon_{F} / k_{\mathrm{B}}$ is the Fermi temperature. By the careful analysis of the downstream spin diffusion length $L_{d}$, we (i) demonstrate that in this regime carrier-carrier interactions become important and (ii) introduce the 'semiconductor degenerate' regime, in which spin-transport properties of a degenerate semiconductor are substantially modified by applying a modest electric field. Finally we discuss how the electric field and Coulomb interaction concur to influence the magnetoresistance of a trilayer system.
\end{abstract}

Spin injection from a ferromagnet into a paramagnetic semiconductor remains one of the challenges in semiconductor spintronics [1]. Recently Yu and Flatté have pointed out [2] that in lightly doped non-degenerate semiconductors the efficiency of spin injection can be highly enhanced by the application of a relatively modest electric field. In addition to enhancing the 'downstream' diffusion length, such an electric field can also substantially reduce the magnetoresistance observed in ferromagnet-semiconductorferromagnet structures [2].

In this work, we analyse spin transport in semiconductors in the regime characterized by $T \lesssim T_{F}$ (intermediate to degenerate), where $T_{F}=\varepsilon_{F} / k_{\mathrm{B}}$ is the Fermi temperature. Such regime is of great importance since it includes the lightly doped semiconductor structures used in most experiments [3].

Following the derivation we proposed in section 5 of [4] and imposing the steady-state condition $\partial\left(\Delta n_{\uparrow}-\Delta n_{\downarrow}\right) / \partial t=$ 0 we obtain the steady-state interacting drift-diffusion equation

$$
\begin{gathered}
-\frac{\left(\Delta n_{\uparrow}-\Delta n_{\downarrow}\right)}{\tau_{s}}+D_{s} \nabla^{2}\left(\Delta n_{\uparrow}-\Delta n_{\downarrow}\right) \\
+\mu_{s} \mathbf{E} \cdot \nabla\left(\Delta n_{\uparrow}-\Delta n_{\downarrow}\right)=0,
\end{gathered}
$$

where $\tau_{s}=\left(1 / \tau_{s f, \uparrow}+1 / \tau_{s f, \downarrow}\right)^{-1}$ is the spin relaxation time, $\mathbf{E}$ the applied electric field, $\Delta n_{\alpha}(\mathbf{r}, t) \equiv n_{\alpha}(\mathbf{r}, t)-n_{\alpha}^{(0)}, n_{\alpha}(\mathbf{r}, t)$ the $\alpha$ density component, $n_{\alpha}^{(0)}$ its equilibrium value and the effective interacting mobility and diffusion constants are given [4], in the paramagnetic case, by $\mu_{s}=e \tau_{D} / m^{*}$ and $D_{s}=\left(\mu_{s} k_{\mathrm{B}} T / e\right)\left(S / S_{c}\right) /\left(1-\rho_{\uparrow \downarrow} / \rho_{D}\right)$. Here $\rho_{D}$ is the ordinary Drude resistivity, $S$ the static longitudinal spin stiffness $\left(S=\partial^{2} f / \partial\left(n_{\uparrow}-n_{\downarrow}\right)^{2}\right.$, with $f$ the free energy per unit volume of a homogeneous interacting electron gas), $S_{c}$ its classical counterpart, and $\rho_{\uparrow \downarrow}$ the spin transresistivity. The spin transresistivity $\rho_{\uparrow \downarrow}(T)$ can be defined as the ratio of the gradient of the electrochemical field for up-spin electrons $\left(E_{\uparrow}\right)$ to the current of down-spin electrons $\left(j_{\downarrow}\right)$ when the current of up-spin electrons is zero:

$$
E_{\uparrow}=\rho_{\uparrow \downarrow}(T) j_{\downarrow}, \quad\left(j_{\uparrow}=0\right) .
$$

$\rho_{\uparrow \downarrow}(T)$ measures the momentum rate exchanged between spinup and spin-down carriers $[4,5]$.

In the paramagnetic limit, Coulomb interactions enter equation (1) through the diffusion constant. We want to point out that the experimentally important regime $T \lesssim T_{F}$ is the same in which Coulomb interactions between carriers of opposite spin can become relevant. They enter $D_{s}$ by affecting the spin stiffness $S$ and generating the spin transresistivity $\rho_{\uparrow, \downarrow}$ $[4,5]:$ for $T \sim T_{F}$ the spin transresistivity has its maximum and can become of the same order as the Drude resistivity [4], 


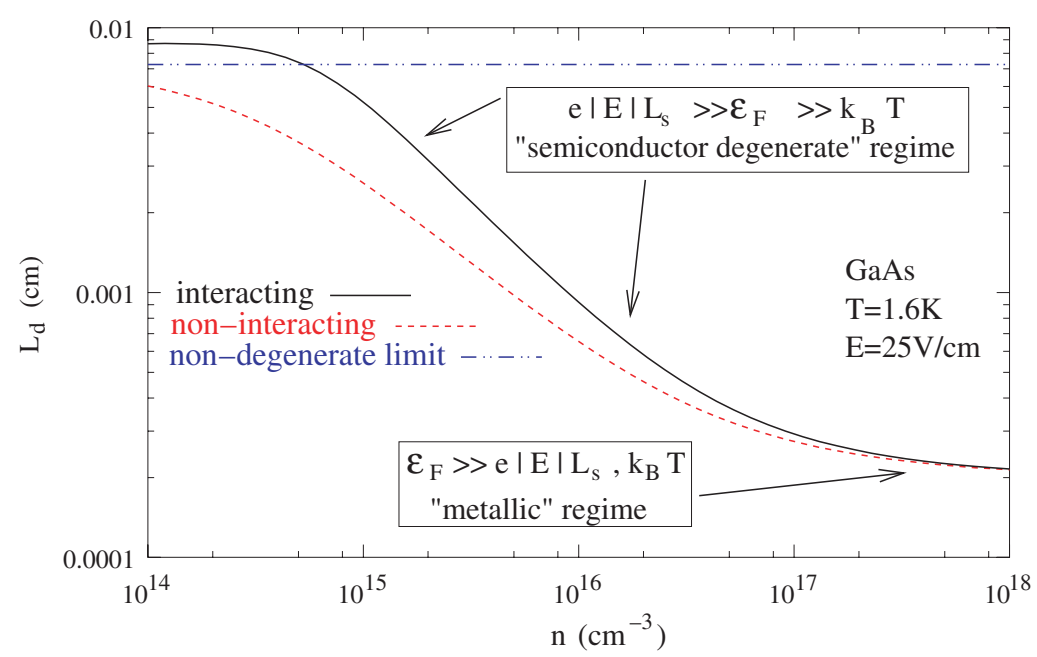

Figure 1. Downstream diffusion length $L_{d}$ versus carrier density. The figure shows that even in the degenerate regime, a modest field can heavily modify $L_{d}$. In the same range of densities the effect of carrier-carrier interaction can more than double the value of $L_{d}$.

while for $T \lesssim T_{F}$ the spin stiffness displays the maximum deviation from its non-interacting approximation [4]. These two combined effects can reduce the non-interacting approximation to $D_{s}$ even by $50 \%$ [4]; according to their $D_{s}$ dependence, Coulomb interaction effects may get amplified as it is the case for the downstream spin-diffusion length (see figure 1).

In one dimension the solution to equation (1) is $\Delta n_{\uparrow}-$ $\Delta n_{\downarrow}=A \mathrm{e}^{x / L_{u}}+B \mathrm{e}^{-x / L_{d}}$ with

$$
L_{u, d}=\left[ \pm \frac{\mu_{s}|E|}{2 D_{s}}+\sqrt{\left(\frac{\mu_{s}|E|}{2 D_{s}}\right)^{2}+\frac{1}{D_{s} \tau_{s}}}\right]^{-1}
$$

the interacting upstream ( $L_{u}$, 'plus' sign) and downstream ( $L_{d}$, 'minus' sign) 'diffusion' lengths. $L_{d}\left(L_{u}\right)$ represents the carrier penetration length along (opposite to) the number current density direction. Again we note that in paramagnets Coulomb interactions enter $L_{u, d}$ through $D_{s}$.

Let us consider a n-doped GaAs sample at $T=1.6 \mathrm{~K}$, and focus on the crossovers undergone by the system when the carrier density is increased keeping the applied electric field fixed. At this temperature, even at very low densities the system is degenerate $\left(\varepsilon_{F} \gg k_{\mathrm{B}} T\right)$, so it is interesting to study the interplay between the Fermi energy and the electric field related energy $e|E| L_{s}$, where $L_{s}=\sqrt{\tau_{s} D_{s}}$ is the spin-diffusion length. As shown in the figure, depending on the system density, the Fermi energy can be low enough to enter, even for moderate electric fields, the 'semiconductor degenerate regime' $e|E| L_{s} \gg \varepsilon_{F}$. The existence of such a regime implies that, even for a degenerate system, the drift term in equation (1) cannot be neglected, i.e. it is not possible to describe spin transport using a diffusion equation for the electrochemical potentials, as usually done for metals [6]. Even in degenerate semiconductor systems in fact, the drift term severely modifies the penetration lengths $L_{u, d}$, varying their values over order of magnitudes (as clearly shown for $L_{d}$ in figure 1). The 'semiconductor degenerate' regime extends to higher densities when the applied field is increased. Note that, even in such regime, the actual value of $L_{d}$ strongly differs from its non-degenerate limit. By increasing the carrier

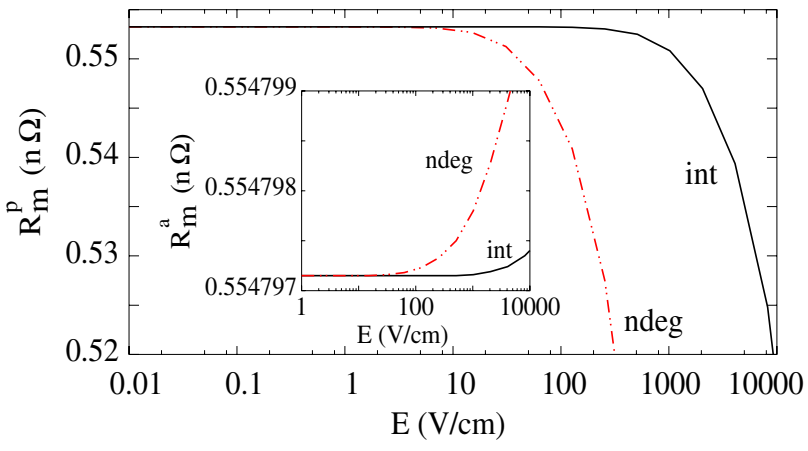

Figure 2. Main panel: magnetoresistance $R_{m}$ for the parallel configuration versus electric field for $p_{f}=0.5$. Its non-degenerate approximation (dashed-double-dot line) is plotted for comparison. Inset: as for main panel, but for the antiparallel configuration.

density, the system will eventually enter the 'metallic' regime (as indicated in the figure), in which the drift term can be neglected and $L_{u, d}$ recover their unperturbed value $L_{s}$.

In figure 1, we plot also the non-interacting approximation to $L_{d}$ (dashed line). As one can see, the correction can be of the order of $100 \%$, definitely non-negligible when quantitative calculations are required. Similarly Coulomb interactions influence the behaviour of the upstream diffusion length $L_{u}$ (not shown here), with the key difference that $L_{u}$ is reduced by Coulomb interactions. Since key quantities such as the spin current polarization and the magnetoresistance are functions of $L_{u}$ and $L_{d}[2,3]$, their values will be consequently affected by electron-electron Coulomb interactions.

As an example, figure 2 illustrates the behaviour of the magnetoresistance $R_{m}$ as a function of the electric field for a ferromagnetic/non-magnetic/ferromagnetic (FM) structure. The non-magnetic material is n-doped GaAs; the parameters are $n=5 \times 10^{16} \mathrm{~cm}^{-3}$, FM-semiconductor conductivity ratio $\sigma^{f} / \sigma_{\mathrm{sc}}=100$, FM spin diffusion length $L_{f}=20 \mathrm{~nm}, L_{s}=2 \mu \mathrm{m}, T=1.6 \mathrm{~K}$ and FM polarization $p_{f}=0.5$. The main panel refers to the configuration in which the two FM magnetizations are parallel. We clearly see that the field decreases the magnetoresistance. The 
opposite trend occurs when the magnetization of one FM is reversed: our calculations show that in the antiparallel case the magnetoresistance is increased by the applied electric field (see inset). In both cases, the non-degenerate approximation (dashed double-dot line) is shown for comparison and it clearly overestimates the field effect. For the carrier density and temperature considered, the Coulomb corrections to the noninteracting approximation are of the order of 20-25\% (not shown).

\section{References}

[1] Schmidt G, Ferrand D, Molenkamp L W, Filip A T and van Wees B J 2000 Phys. Rev. B 62 R4790

Rashba E I 2002 Preprint cond-mat/0206129

[2] Yu Z G and Flatté M E 2002 Phys. Rev. B 66235302

[3] D'Amico I 2003 Preprint cond-mat/0306544

[4] D'Amico I and Vignale G 2002 Phys. Rev. B 65085109

[5] D'Amico I and Vignale G 2000 Phys. Rev. B 624853

[6] van Son P C, van Kempen H and Wyder P 1987 Phys. Rev. Lett. 582271 